\title{
SIMULATION OF DYNAMIC STABILITY PERFORMANCE OF TRAFFIC SAFETY BRIDGE TOWER DETECTOR SYSTEM
}

\author{
Wu Lei ${ }^{1}$, Viet Hung Luu ${ }^{2}$, Shahreen Kassim ${ }^{3}$ \\ ${ }^{1}$ Automotive Engineering Department, Zibo Vocational institute Shandong, Zibo 253314, China \\ ${ }^{2}$ Maritime College No. 1, Haiphong, Vietnam \\ ${ }^{3}$ Faculty of Computer Science and Information Technology, Universiti Tun Hussein Onn Malaysia \\ Email:wleiu12031@126.com
}

\begin{abstract}
The study is to accurately simulate the actual motion of the bridge tower detector to provide a virtuous method for the bridge safety detection process. This paper takes the bridge tower detector as the research object and used UG three-dimensional modeling software to establish a 3D solid model of the virtual prototype of the bridge tower detector. The model is imported into the Automatic Dynamic Analysis of Mechanical Systems (ADAMS) environment through Parasolid format to verify the accuracy of the data transmitted. On the premise of referring to similar studies, combining the actual situation of the bridge tower detector studied, the macro function provided by ADAMS is used to establish the wire rope system model of the bridge tower detector. In the process of simulating the vibration of the bridge tower detector, there has been vibration throughout the operating phase. The large fluctuations due to system instability at startup were ignored which almost fluctuated between $-250 \mathrm{~mm} / \mathrm{s}^{2}$ to $250 \mathrm{~mm} / \mathrm{s}^{2}$ acceleration. The fluctuations show periodic changes, and a large fluctuation occurs about every 7 seconds, which is directly related to the characteristics of the rope. In the process of simulating the bridge tower detector attitude, the rotation angle of the detector relative to the center of mass coordinates in the direction of roll degree of freedom fluctuates between $0.01^{\circ}$ to $0.05^{\circ}$. The angular velocity varies between $-0.025 \mathrm{deg} / \mathrm{s}$ and $0.025 \mathrm{deg} / \mathrm{s}$, which is almost negligible. The designed bridge tower detector has a decent overall operating condition and can encounter the requirements for future studies. This study can provide theoretical support for the actual operation of the bridge tower detector.
\end{abstract}

Keywords: Bridge Tower Detector, System Dynamics, ADAMS, Simulink, Bridge Safety.

\section{Introduction}

With the rapid development of science and technology, bridge technology is becoming more mature and progressive. Especially in recent years, traffic development in China has been rapid, and bridge construction has risen to unprecedented heights [1]. The development of the bridge cause makes the mileage of bridges constantly increase, and it will inevitably accompany the increase in traffic volume. Increasingly large overloaded vehicles have brought severe tests on the traffic capacity and operational safety of highways and bridges. At the same time, some bridges have become increasingly damaged due to years of disrepair, and their aging speeds are more difficult to meet today's traffic capacity requirements. The contradictions caused by the mentioned two phenomena have become increasingly prominent [2]. According to surveys, there are currently more than 90,000 dangerous bridges in China, and the situation of low load standard is also very serious.
Although some old and dangerous bridges have been rebuilt, there are still a large number of dangerous bridges that remain with tribulation, which has seriously affected traffic safety [3]. Therefore, to better ensure the safety and smoothness of traffic, and to ensure the safe driving of vehicles, the work of strengthening the detection and maintenance of bridges must be the focus of future work by relevant scientific researchers and traffic management departments at all levels. Only by taking precautions we can ensure that the bridge is always in good working condition [4].

To accurately simulate the actual motion of the bridge tower detector, a more complete virtual prototype model is needed. Automatic Dynamic Analysis of Mechanical Systems (ADAMS) focuses on mechanical dynamic simulation, but the control is relatively lacking [5-6]. In addition, the algorithms that come with ADAMS software are often inadequate when solving complex virtual prototype models. At this time, the most effective method is to seek the assistance of third-party professional 
control software. As one of the important toolboxes of MATLAB, Simulink occupies an important position in the field of professional control and designing aid. Because of its powerful solving function, Simulink can quickly solve differential equations, can simulate various dynamic systems in real-time, with relatively small time consumption and errors [7]. The method gives full play to the mechanical dynamic analysis capabilities of ADAMS and makes use of Simulink's powerful solution capabilities to make up for the shortcomings of ADAMS in solving complex models. Compared with the traditional method, it has the characteristics of accuracy, convenience, and speed, which can help some complex mechanical systems to perform dynamic simulations smoothly and effectively. Some scholars used the joint simulation of ADAMS and Simulink to accurately study the multi-body dynamics model of the vehicle model integrated with air springs [8-9]. Some scholars have proposed a law on the motion of servo motors to obtain the kinematic relationship of flexible motion between the motor and the connector on the robot arm. Similarly, the feasibility of the control and actuator system is verified by the joint simulation of ADAMS and Simulink [10].

This paper takes the bridge tower detector as the research object and uses the three-dimensional modeling software UG and virtual prototype dynamic analysis software ADAMS to establish the bridge tower detector model. Then, combined with the control system simulation software MATLAB/Simulink, a mechatronics joint simulation model is established. Also, its motion stability is studied and analyzed and ultimately contributes to the detection of bridge safety. The novelty of this paper is to combine ADAMS and MATLAB/Simulink in the simulation research of bridge tower detector, reducing the experimental cost.

\section{Method}

The structure of the bridge tower detector is complex, and its stable operation directly affects the detection and position accuracy of the bridge detection equipment. Through theoretical calculations and experimental studies, the factors which affect these dynamic performances and the methods of improvement are analyzed to rationally improve and optimize the structure and parameters of the equipment [11-12].

Since the bridge tower detector is a new type of testing equipment, there is no related research on it at present. For the research of improving the dynamic performance of such equipment, experimental methods are generally used. Because the most real and effective data can be obtained through experiments. But the experiment can only be implemented after the product prototype is produced, which increases the risk of product design errors, cost, and development cycle. If the virtual prototype technology is used, it can not only modify in time to reduce design errors but also speed up the product development cycle and effectively reduce the development funds.

\subsection{Joint simulation method}

All required software to draw the work are illustrated in flowchart Figure 1. First, a threedimensional solid model of the virtual prototype of the bridge tower detector is established by using the three-dimensional design software UG, and it is imported into the dynamic analysis software ADAMS. Then, in the ADAMS software, the ADAMS macro command is used to establish the wire rope system model of the bridge tower detector. A tireroad coupling model is established using the tire and road attribute files. In accordance with the kinematic relationship and motion of the physical prototype, the drives, constraints, and forces are added to each component in the ADAMS software. A motor control model is built with MATLAB/Simulink. According to the theory of mechatronics and combining the Control module in ADAMS, the joint simulation model of the bridge tower detector is completed, to perform the dynamic analysis of the whole machine.

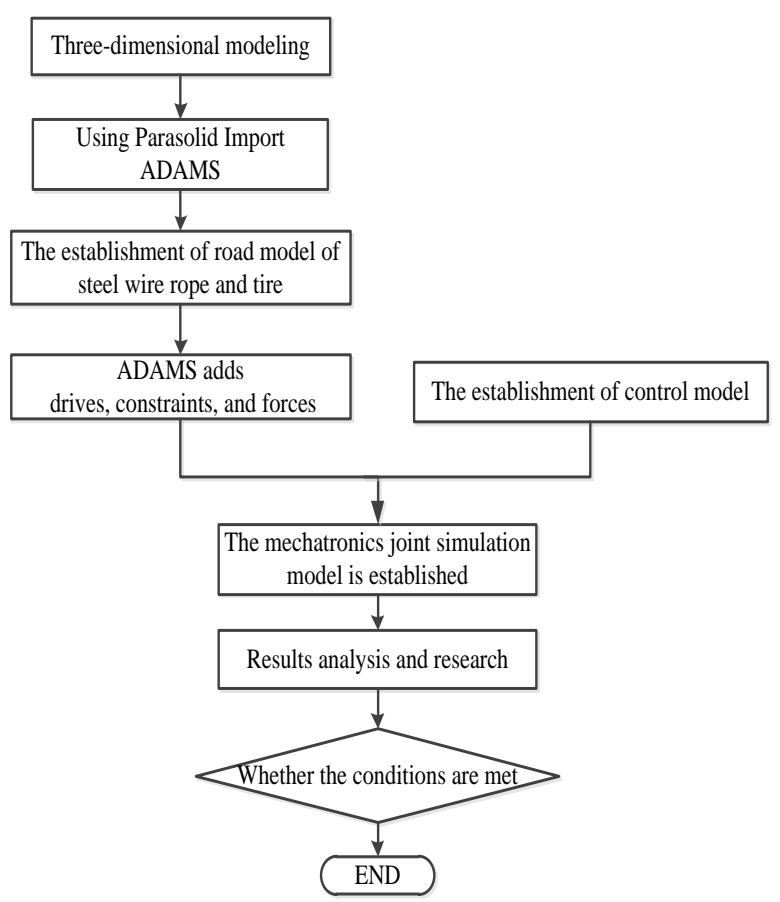

Figure 1: Mechatronics joint simulation flowchart

\subsection{ADAMS modeling method}

ADAMS software is a virtual prototype analysis software developed by Mechanical Dynamics Company, and widely used virtual prototype analysis software. It can study the maximum load of components and be used to analyze the antideformation capability of the structure and dynamic characteristics such as stress changes, shape 
displacements and strain states under load condition [13].

In ADAMS, components can be divided into rigid components and flexible components. When a force is applied to a component, if the geometry will not change at any specified time, such elements are called rigid elements, but has the mass and inertia attribute. In contrast, deformed elements are called flexible elements. Multi-body dynamics is the premise of research on complex motion software using ADAMS software. The computer simulates the virtual prototype and obtains its performance indicators, helping developers understand whether there are defects in the design of the virtual prototype and make up for the defects. It not only significantly reduces product development time, but also decreases research and development costs [1415].

The mechanical system simulation analysis provided by ADAMS can effectively reflect the actual system motion and meet the application standards, making ADAMS the most widely used mechanical system motion simulation analysis software in the world. Before the designed product is produced, the user only needs to simulate and analyze its virtual prototype to predict its performance in advance, that is, to achieve the purpose of timely modification and optimization. Therefore, this software got more and more attention from relevant fields worldwide. The ADAMS simulation flowchart is shown in Figure 2.

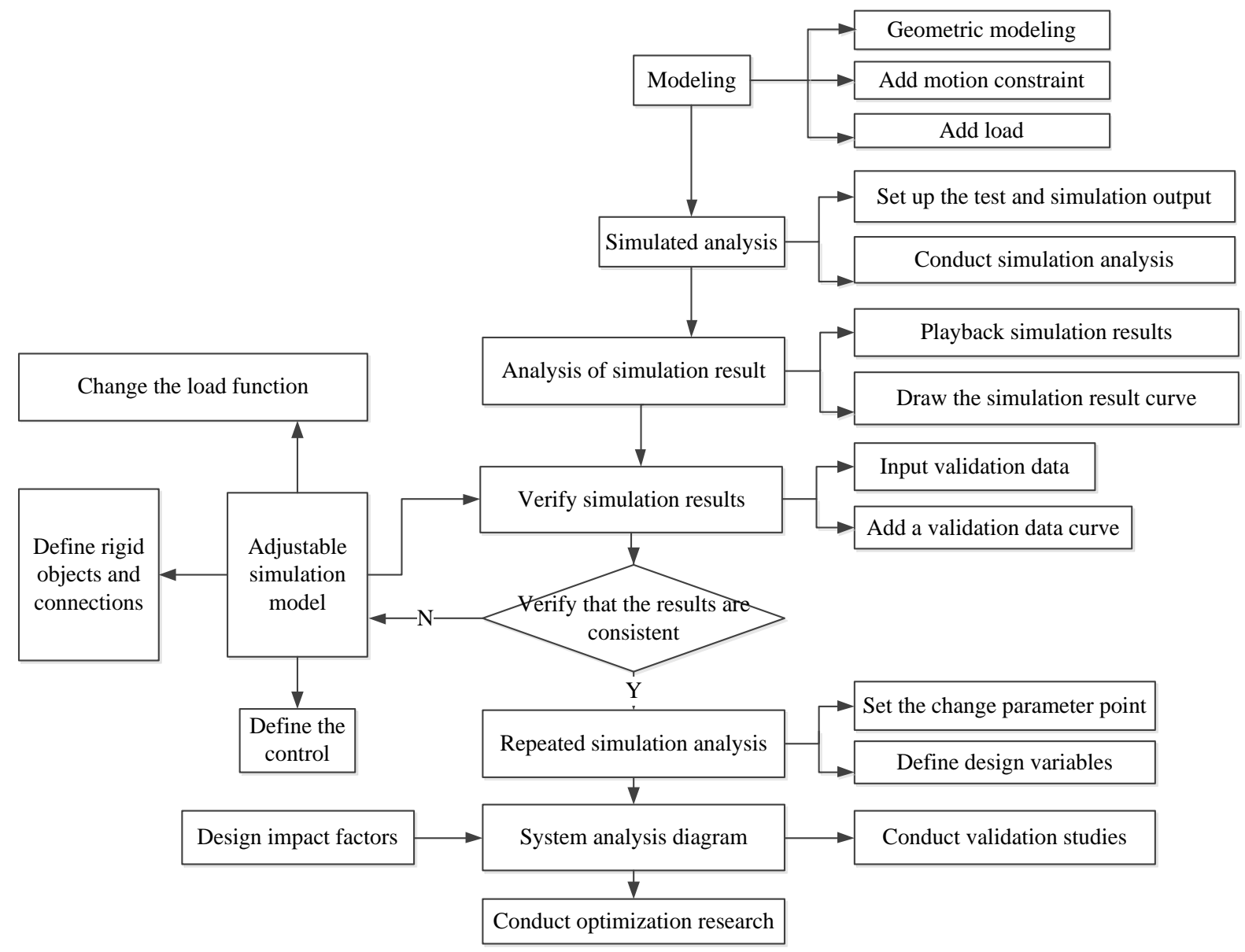

Figure 2: ADAMS simulation flowchart

\subsection{Virtual prototype design}

The emergence of virtual prototype technology is undoubtedly a major revolution for the traditional product development model. For the traditional product development model, as shown in Figure 3, the development process begins with product design, and a physical prototype is manufactured based on the design information. After the prototype is manufactured, the prototype is tested and evaluated by experiments and other theoretical manners.

Then, according to the comparison between the evaluation data and the original design data, a certain optimization is made to the original design, which will inevitably extend the product development cycle. Moreover, this design pattern will have a physical prototype manufactured and modified once every cycle. Therefore, the increase in development costs is also inevitable. 


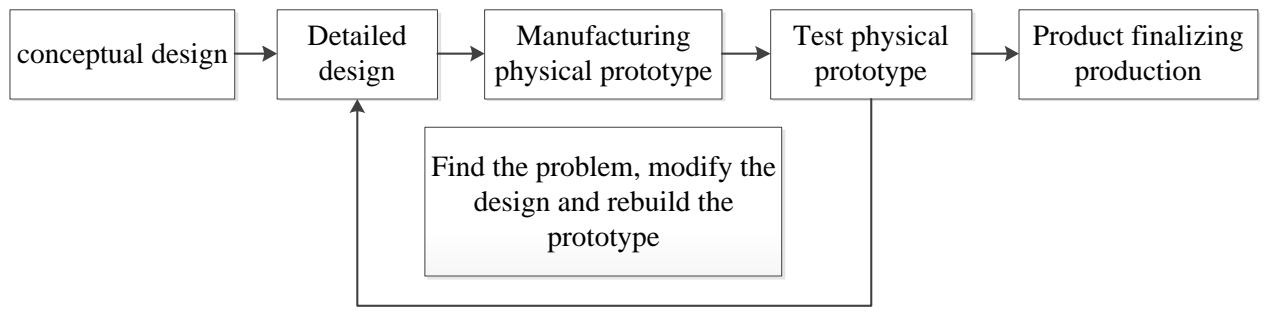

Figure 3: Traditional product development process

Obviously, for the increasingly competitive modern market, this model cannot keep up with the pace of the times, and sooner or later it will be eliminated. The following alternative technology is the virtual prototype development technology.

As shown in Figure 4, because there is no need to manufacture a physical prototype, the new technology shortens the research and development cycle and produces high-quality new products in the shortest time, greatly reducing product development costs. Therefore, it is possible to respond quickly to the changing market.

From the development flowchart, the huge advantages of using the virtual prototype technology development process are more intuitively seen.

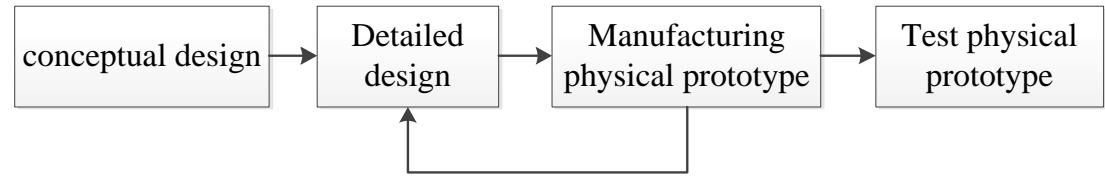

Figure 4: Traditional product development process

The prototype model is established by 3D modeling software and assembled based on the relationship of position and constraints. Then, it needs to be transmitted to the ADAMS environment through a certain format. The wire rope system model and the tire-road coupling model are established through the related functions of ADAMS.

The constraints and forces are added to connect the various components.
Therefore, it is important to add elements such as drives, constraints, and forces between components based on the actual motion of the physical prototype. But it is not the last step because some errors will inevitably occur during the modeling process.

Therefore, it is necessary to constantly check and verify the model, find out error and timely modify it, to build a complete and correct prototype model. The modeling flowchart is shown in Figure 5.

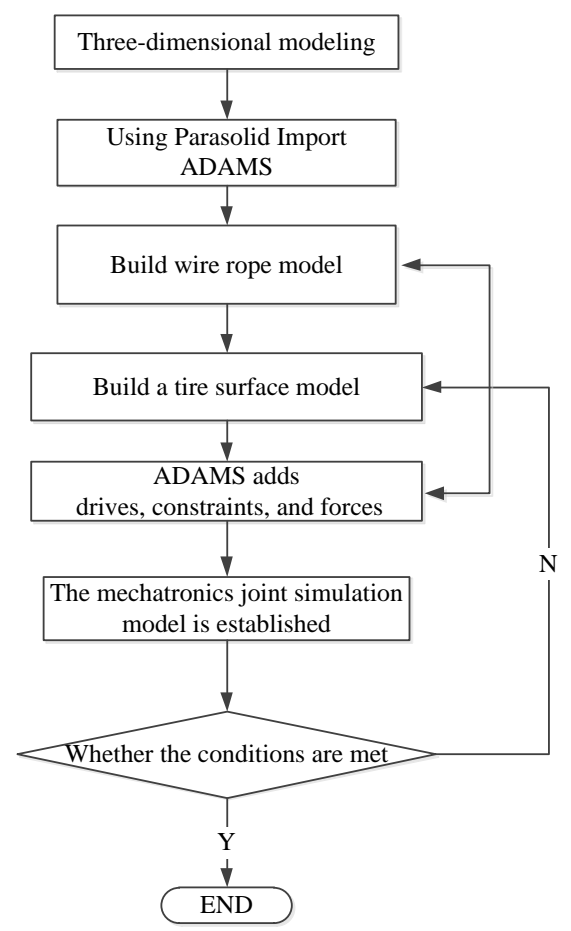

Figure 5: Modeling flowchart of the virtual prototype of the bridge tower detector 


\subsection{Simulink motor simulation}

Model of the asynchronous motor on any twophase rotating coordinate system $d, q, 0$ : The $d, q, 0$ system is based on the double reaction principle. The $\mathrm{d}$ axis coincides with the rotor direct axis. The $\mathrm{q}$ axis is 90 degrees coordinate system ahead of the $d$ axis in the same direction as the rotor and rotates at synchronous speed. Whereas, abc is a representation of three-phase voltage. Three-phase AC power source is composed of three AC potentials with the same frequency, equal amplitude, and a phase difference of 120 degrees. Since the d, q, 0 system can directly reflect the rotation angle and position of the rotor and the stator, the $\mathrm{d}, \mathrm{q}, 0$ system is used in this paper to facilitate the solution. The stator of a wound asynchronous motor has three-phase symmetrical windings of $\mathrm{A}, \mathrm{B}$, and $\mathrm{C}$. The rotor has three-phase symmetrical windings of $a, b$, and $c$. According to the equation of coordinate transformation, the voltage, current, and magnetic flux of the stator and rotor are transformed to the d, $\mathrm{q}, 0$ coordinate system. Then, the mathematical model of the asynchronous motor on any two-phase rotating coordinate system is obtained. The mathematical model has only two phases and can directly reflect the rotation angle and position of the rotor and stator, which is simpler than the original. Because its coordinate system is arbitrarily rotated, it is more general farm.

The three-phase asynchronous motor simulation model is composed of four modules: stator model, rotor model, magnetic flux model, and torque model. According to the voltage, magnetic flux and torque equation of the stator and rotor of the motor under the $\mathrm{d}, \mathrm{q}, 0$ system, a simulation model of the stator, rotor, magnetic flux and torque of the motor can be established.

\section{Results and Discussion}

\subsection{Simulation results of bridge tower detector vibration}

Considering the configuration of the computer, this project only considers the simulation lifting once, and the time is 35 seconds. Using a trapezoidal speed chart, the time distribution of acceleration, uniform speed, and deceleration are respectively 5 seconds, 25 seconds, and 5 seconds, with a constant speed of $500 \mathrm{~mm} / \mathrm{s}$. Before the joint simulation, to verify whether the model can run normally, the STEP function is used to control the motor speed instead of Simulink. Among them, the STEP function is a design process function, which can display the law of motion of the drive with time in the design, and its format is STEP (time, $t 1, x 1, t 2, x 2$ ). Where: $t 1$ is the start time, and $\mathrm{x} 1$ is the initial value. While, $\mathrm{t} 2$ is the end time, and $\mathrm{x} 2$ is the end value.
There are two adjusting springs on both sides of the detector, which are used to adjust the force between the detector and the side wall of the bridge tower as well as act as the contact force between the guide wheel and the guide wire rope. Also, they can also play a role in damping. The initial parameters of the adjustment springs set on both sides in ADAMS: $\mathrm{Kc}=11 \mathrm{~N} / \mathrm{mm}, \mathrm{Cc}=2.3 \mathrm{~N} \bullet \mathrm{s} / \mathrm{mm}$.

There has been vibration throughout the operating stage and large fluctuations due to system instability at start-up are ignored. The vibration acceleration of the entire lifting process almost fluctuates between $-250 \mathrm{~mm} / \mathrm{s} 2$ to $250 \mathrm{~mm} / \mathrm{s} 2$. The fluctuations show periodic changes, and a large fluctuation occurs about every 7 seconds, which is related to the characteristics of the rope.

The vibration of the detector's center of mass in the $\mathrm{X}$ and $\mathrm{Y}$ directions exists throughout the entire lifting process. But overall, the whole vibration process is relatively stable. The maximum value of the vibration acceleration of the center of mass in the $\mathrm{X}$ direction was $280.92 \mathrm{~mm} / \mathrm{s}^{2}$, whereas the maximum value of the vibration acceleration in the $Y$ direction was $636.16 \mathrm{~mm} / \mathrm{s}^{2}$.

There are many reasons for the large fluctuations in lifting acceleration phenomenon. The main reason is that the wire rope model is simulated by many small cylinders, not a flexible body in the true sense. When the small cylinder rises to the position of the motor drum, there must be a collision and impact with the motor drum, which will inevitably lead to acceleration fluctuations in the lifting direction. In addition, parameters such as the stiffness and damping of the wire rope as well as errors in the modeling of the detector model will cause the acceleration curve of the bridge tower detector to deviate from the theoretical curve during operation.

\subsection{Simulation results of attitude stability of bridge tower detector}

The peak values due to system instability in the first few seconds of the simulation are ignored. From the information shown in Figure 6, the maximum value of the beating displacement of the detector's center of mass in the direction of surge degree of freedom is $4.58 \mathrm{~mm}$. The maximum beating displacement of the detector's center of mass in the direction of sway degree of freedom is $7.51 \mathrm{~mm}$. According to the displacement curve in the direction of heave degree of freedom, it can be known that the stroke at the acceleration phase in the first 5 seconds is about $1248 \mathrm{~mm}$. The stroke in the middle 25 seconds at constant speed phase is about $12538 \mathrm{~mm}$. The stroke during the deceleration phase of the next 5 seconds is also about $1252 \mathrm{~mm}$. Therefore, the total lifting stroke is about $15000 \mathrm{~mm}$, which reaches the designed stroke. 


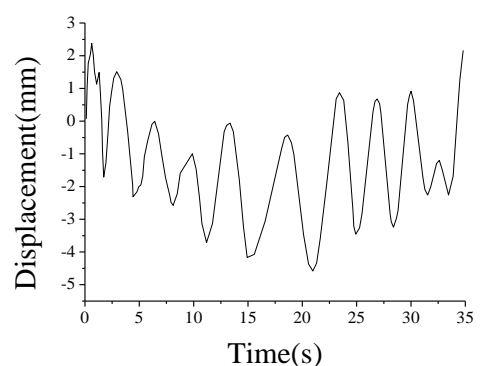

A

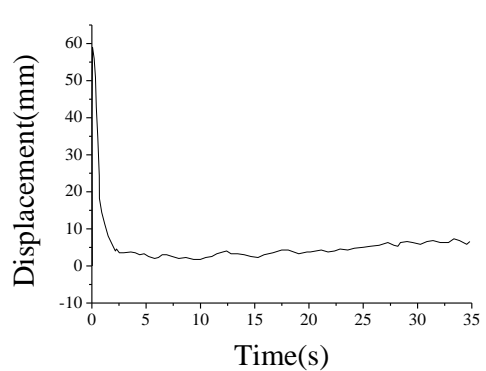

$\mathrm{B}$

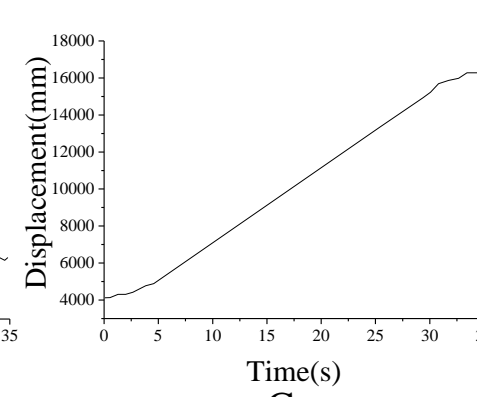

$\mathrm{C}$

Figure 6: Displacement of the detector's center of mass in various degrees of freedom (A: Surge; B: Sway; C: Heave)

The rotation angles and angular velocities in three degrees of freedom of the pitch, yaw, and roll of the detector relative to the center of mass coordinates

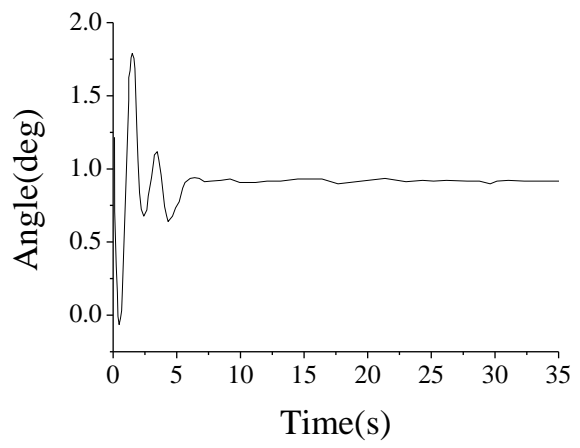

A were taken as the research objects. The obtained curves are shown in Figures 7-9. For easy identification, the angle unit is expressed as ${ }^{\circ}$.

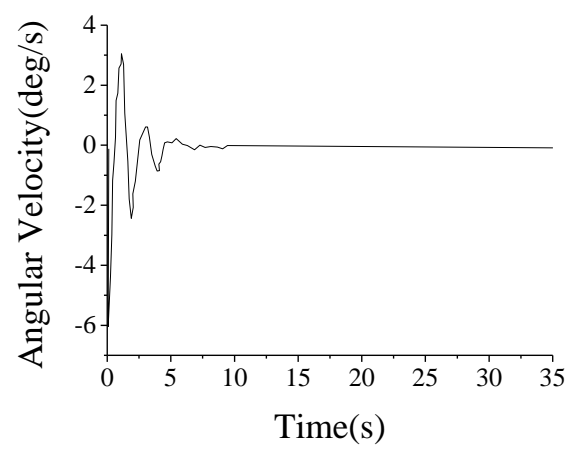

$\mathrm{B}$

Figure 7: Rotation angle and angular velocity of the detector relative to the center of mass in the direction of pitch degree of freedom

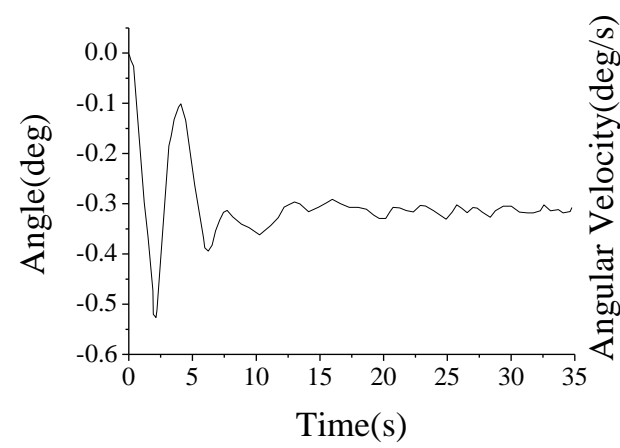

A

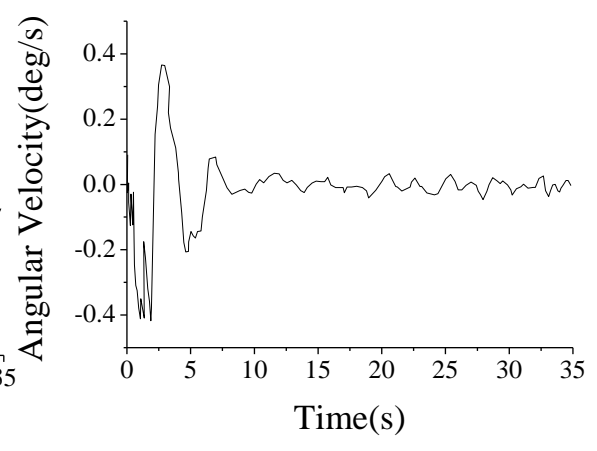

$\mathrm{B}$

Figure 8: Rotation angle and angular velocity of the detector relative to the center of mass in the direction of yaw degree of freedom

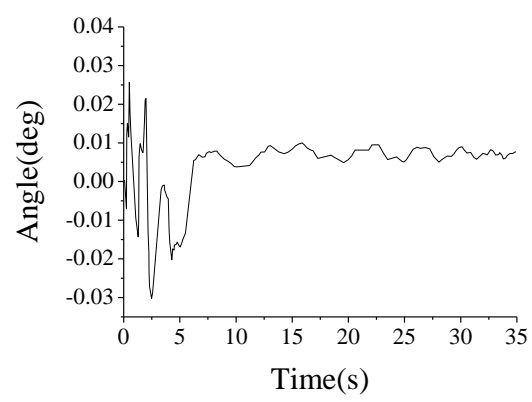

A

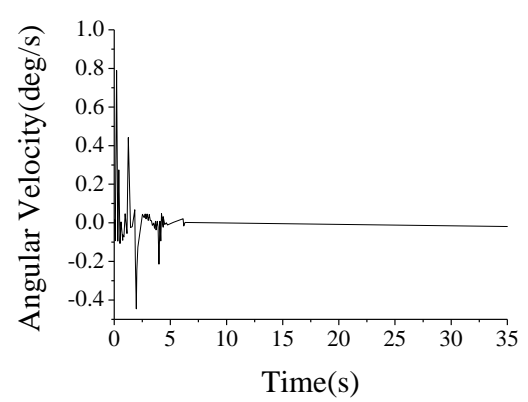

$\mathrm{B}$

Figure 9: Rotation angle and angular velocity of the detector relative to the center of mass in the direction of roll degree of freedom 
From Figure 7, during the entire stroke, the rotation angle of the detector relative to the center of mass in the direction of pitch degree of freedom is almost stable at about $0.9^{\circ}$ except for the large fluctuation at the beginning of the simulation. The angular velocity change trend is consistent with the angle change, and the fluctuation is small. Figure 8 shows that during the entire stroke, the rotation angle of the detector relative to the center of mass coordinate in the direction of yaw degree of freedom fluctuates about $0.3^{\circ}$, and the maximum does not exceed $0.6^{\circ}$. The angular velocity fluctuates between $-0.05 \mathrm{deg} / \mathrm{s}$ to $0.05 \mathrm{deg} / \mathrm{s}$ with lesser variations. Figure 9 shows that during the entire stroke, the rotation angle of the detector relative to the center of mass in the direction of roll degree of freedom fluctuates about $0.01^{\circ}$, and the maximum does not exceed $0.05^{\circ}$. The angular velocity fluctuates between $-0.025 \mathrm{deg} / \mathrm{s}$ and $0.025 \mathrm{deg} / \mathrm{s}$, which is almost negligible. From the above analysis, under the condition that the vibration acceleration of the detector meets the relevant national standards of the vertical elevator, its motion morphology during the entire stroke is also maintained well, which ensures that it can successfully complete the detection.

\section{Discussion}

According to the principle of joint simulation of ADAMS and Simulink, this paper processes the definition of input variables and output variables of the model. The Simulink control model and the mechanical subsystem model are connected to form the final mechatronics simulation model. Before conducting the joint simulation, the model is firstly simulated by using the STEP function to control the motor speed. Also, the defects of the overturning of the model are found and corresponding improvement measures are taken. The necessity of mechatronics joint simulation is verified. Some scholars have proposed a law on the motion of servo motors to obtain the kinematic relationship of flexible motion between the motor and the connector on the robot arm. Also, the feasibility of the control and actuator system is verified by the joint simulation of ADAMS and Simulink method. It is almost the same as the research idea of this paper, but the research direction is different [16-17]. The research in this paper can almost monitor the motion state of the bridge tower detector, which is in line with the experimental purpose and experimental goal.

In the process of simulating the vibration of the bridge tower detector, there has been vibration throughout the operating phase. The large fluctuations due to system instability at start-up are ignored. The vibration acceleration of the entire lifting process almost fluctuates between $250 \mathrm{~mm} / \mathrm{s} 2$ to $250 \mathrm{~mm} / \mathrm{s} 2$. The fluctuations show periodic changes, and a large fluctuation occurs about every 7 seconds, which is related to the characteristics of the rope. In the process of simulating the bridge tower detector attitude, the rotation angle of the detector relative to the center of mass coordinates in the direction of roll degree of freedom fluctuates around $0.01^{\circ}$, and the maximum value does not exceed $0.05^{\circ}$. The angular velocity fluctuates between $-0.025 \mathrm{deg} / \mathrm{s}$ and $0.025 \mathrm{deg} / \mathrm{s}$, which is almost negligible.

This paper studies the structure and operation mechanism of the bridge tower detector and uses UG to establish a three-dimensional solid model of the virtual prototype of the bridge tower detector. The model is imported into the ADAMS environment through the Parasolid format to verify the accuracy of the data transmitted in Parasolid format. On the premise of referring to similar studies, combining the actual situation of the bridge tower detector studied in this subject, the macro function provided by ADAMS is used to establish the wire rope system model of the bridge tower detector.

\section{Conclusions}

In this paper, the three-dimensional modeling software UG is used to study the bridge tower detector, and the virtual prototype dynamic analysis software ADAMS is used to establish the bridge tower detector model. Then, combined with the control system simulation software MATLAB/Simulink, a mechatronics joint simulation model is established, and its motion stability is studied and analyzed, which contributes to the detection of bridge safety. The joint simulation method is used to simulate the final model, and the vibration acceleration of the detector is analyzed. With reference to the stability evaluation method of the vertical elevator, the model is analyzed and evaluated. Also, the relevant parameters affecting its vibration are optimized so that the vibration acceleration of the detector meets the reference standard. In addition, the attitude stability of the detector is studied and analyzed, as well as the analysis of the impact of the wire rope performance on the system is performed. The results show that the designed bridge tower detector is in good overall operating condition and can meet the requirements for use. The feasibility of the application of the mechatronics joint simulation technology to the lifting system of complex models is verified.

The research in this paper has achieved some useful results. However, due to the limited academic level and ability of the author, the paper still has the following deficiencies, which need to be studied and improved in future work. From the perspective of dynamic modeling, since the bridge tower detector is a complex multi-degree-of-freedom coupling system, the vibration of the wire rope will also affect the operation stability of the detector. At the same time, adjusting the time-variant characteristics of the 
parameters of the spring, the relevant dynamic parameters of the running tire, as well as the actual wire rope's mass, stiffness, damping, and other parameters will all affect the whole machine system to varying degrees. The in-depth study of the relationship between the system parameters and the vibration characteristics of the bridge tower detector as well as the establishment of a more accurate dynamic model are significant for improving the operation stability of the detector. Generally, there is the pressure of wind present at the height of the bridge tower, and the existence of wind load will inevitably affect the operation of the detection equipment. But this paper does not analyze the operation stability of the bridge tower detector under wind load. Therefore, the design and analysis of the bridge tower detector combined with aerodynamics can better improve its operating stability.

\section{References}

[1] Wang R, Xu Y, Li J. Transverse seismic behavior studies of a medium span cable-stayed bridge model with two concrete towers. Journal of Earthquake Engineering, 2017, 21(1), pp. 151168.

[2] Yi J, Li J, Guan Z. Shake Table Studies on Viscous Dampers in Seismic Control of a Single-Tower Cable-Stayed Bridge Model under Near-Field Ground Motions. Journal of Earthquake and Tsunami, 2018, 12(05), pp. 1850011.

[3] Ovett M, Chorzepa M G, Durham S, et al. Vulnerability to failure of cable-stayed bridges for beyond-design basis wind events. Engineering Failure Analysis, 2018, 91, pp. 182200.

[4] Kang H J, Guo T D, Zhao Y Y, et al. Dynamic modeling and in-plane 1: 1: 1 internal resonance analysis of cable-stayed bridge. European Journal of Mechanics-A/Solids, 2017, 62, pp. 94-109.

[5] Fabbrocino F, Modano M, Farina I, et al. Optimal prestress design of composite cable-stayed bridges. Composite Structures, 2017, 169, pp. 167-172.

[6] S.K. Gugulothu, N. Prabhu Kishore, V. Phani Babu, Girish Sapre (2019). Cfd Analysis on Different Piston Bowl Geometries by Using Split Injection Techniques. Acta Mechanica Malaysia, 2(1): 23-28.
[7] $\mathrm{Li} \mathrm{H}, \mathrm{Ou} \mathrm{J}$. The state of the art in structural health monitoring of cable-stayed bridges. Journal of Civil Structural Health Monitoring, 2016, 6(1), pp. 43-67.

[8] Xiong W, Dong X, Tang $\mathrm{P}$, et al. Identification Method for Pylon Scour Depth of Cable-stayed Bridges by Tracing Dynamic Index. Hunan Daxue Xuebao/Journal of Hunan University Natural Sciences, 2017, 44(11), pp. 145-155.

[9] Kadhum Audaa Jehhef and Mohamed Abed Al Abas Siba (2019). Effect Of Surfactant Addition On The Nanofluids Properties: A Review. Acta Mechanica Malaysia, 2(2): 1-19, DOI: 10.26480/amm.02.2019.01.19

[10] Nazarian E, Ansari F, Zhang X, et al. Detection of tension loss in cables of cable-stayed bridges by distributed monitoring of bridge deck strains. Journal of Structural Engineering, 2016, 142(6), pp. 04016018.

[11] Om Prakash Singh, Gaurav Kumar, Mukesh Kumar (2019). Role of Taguchi And Grey Relational Method in Optimization of Machining Parameters of Different Materials: A Review. Acta Electronica Malaysia, 3(1): 19-22.

[12] Mao J X, Wang H, Fu Y G, et al. Automated modal identification using principal component and cluster analysis: Application to a long-span cable-stayed bridge. Structural Control and Health Monitoring, 2019, 26(10), pp. e2430.

[13] Han Q, Wen J, Du X, et al. Simplified seismic resistant design of base isolated single pylon cable-stayed bridge. Bulletin of Earthquake Engineering, 2018, 16(10), pp. 5041-5059.

[14] Farah Ruzanna Ridzuan, Hairulnizam Mahdin, Shahreen Kasim, Mohd Sanusi Azmi (2019). An Image-Based Captcha System Using Click. Acta Electronica Malaysia, 3(1): 23-25.

[15] Sun M, Makki Alamdari M, Kalhori $H$. Automated operational modal analysis of a cable-stayed bridge. Journal of Bridge Engineering, 2017, 22(12), pp. 05017012.

[16] Bedon C, Dilena M, Morassi A. Ambient vibration testing and structural identification of a cable-stayed bridge. Meccanica, 2016, 51(11), pp. 2777-2796.

[17] Neelam Gupta, Neel Kanth (2019). Analytical Approximate Solution Of Heat Conduction Equation Using New Homotopy Perturbation Method. Matrix Science Mathematic, 3(2): 0107. 